\title{
Two Types of Extended Liver Resection for Advanced Gallbladder Cancer: How to Do It
}

\author{
Satoshi Kondo ${ }^{\dagger}$ Satoshi Hirano Eiichi Tanaka Takahiro Tsuchikawa \\ Kentaro Kato Jo Matsumoto Yuya Nasu Toshiaki Shichinohe \\ Department of Surgical Oncology, Hokkaido University Graduate School of Medicine, Sapporo, Japan
}

\section{Key Words}

Right hepatectomy $\cdot$ Biliary reconstruction $\cdot$ Portal reconstruction $\cdot$ Ventral hepatectomy $\cdot$ Para-aortic lymphadenectomy

\section{Abstract \\ Background: Extended liver resection is necessary for ad- vanced gallbladder cancer with hepatic involvement to achieve Ro resection. However, its type or extent and its sur- gical technique have yet to be established. Methods: To ex- clude systemic disease, frozen section biopsy following sys- tematic para-aortic lymphadenectomy is recommended be- fore starting extended surgery with curative intent because para-aortic nodes are involved more frequently than expect- ed. Right hepatectomy with biliary reconstruction should be indicated for the hepatic hilum type of advanced gallbladder cancer in which a relatively small tumor in the gallbladder neck infiltrates the hepatic hilum and causes obstructive jaundice. Ventral hepatectomy without biliary reconstruc- tion can be applied for the hepatic bed type in which a large mass in the gallbladder fundus and body penetrates into the hepatic parenchyma through the gallbladder bed. Results:}

The two types of extended liver resection were successfully performed in representative cases. Detailed procedures were described. Long-term survival without disease recurrence has been achieved in both cases. Conclusion: The type of extended liver resection should be chosen according to the mode of tumor spread in advanced gallbladder cancer without distant metastasis.

Copyright $\odot 2011$ S. Karger AG, Basel

\section{Introduction}

Although advanced gallbladder cancer is associated with dismal prognosis, extended surgery to achieve R0 resection may bring long-term survival in selected patients as long as distant metastasis is absent $[1,2]$. Since advanced gallbladder cancer often involves the liver, the need for extended liver resection is highlighted for radical surgery with curative intent. In the present article, indication for extended surgery and mode of tumor spread are discussed as prerequisite information; then, the two types of extended liver resection are demonstrated with presentation of representative cases.

\section{KARGER}

Fax +4161306 1234

E-Mail karger@karger.ch

www.karger.com (c) $2011 \mathrm{~S}$. Karger AG, Basel

$0253-4886 / 11 / 0282-0148 \$ 38.00 / 0$

Accessible online at:

www.karger.com/dsu
Prof. Satoshi Hirano

Department of Surgical Oncology, Division of Surgery

Hokkaido University Graduate School of Medicine

North-15 West-7, Kita-ku, Sapporo 060-8638 (Japan)

Tel. +81 11706 7714, Fax +81 11706 7158, E-Mail satto@med.hokudai.ac.jp 


\section{Indication for Extended Surgery for Advanced Gallbladder Cancer}

Extended surgery for advanced gallbladder cancer should be indicated only in local or regional disease because it is associated with considerable postoperative morbidity [3], and systemic disease with distant metastasis cannot be cured by surgical intervention. The positive rate of the para-aortic lymph nodes proved $38 \%$ in a previous study of 60 patients with advanced gallbladder cancer who underwent radical resection and routine regional and para-aortic lymphadenectomy [4]. The study concluded that regional and para-aortic lymphadenectomy provided no survival benefit for patients with para-aortic disease, which had an influence on poor prognosis equivalent to that of distant metastasis such as hepatic or peritoneal deposits. Therefore, any metastatic lesion including paraaortic disease should not be missed in preoperative workup; however, positive predictive values of para-aortic lymph node metastasis by CT evaluation ranged from 13 to $36 \%$ (extremely low) [5]. Even staging laparoscopy cannot identify para-aortic metastasis, unlike hepatic or peritoneal metastasis. A sampling biopsy of the para-aortic nodes before starting extended curative surgery is recommended because they are involved more frequently than expected. Practically, it is included in our departmental policy that extended surgery with curative intent should be converted into a palliative procedure or exploration alone when frozen section biopsy following systematic para-aortic lymphadenectomy has shown any positive node.

\section{Mode of Tumor Spread}

The directions of local spread in advanced gallbladder cancer vary as direct hepatic invasion, duodenal and/or colonic involvement, and biliary infiltration within the hepatoduodenal ligament. Basically, the modes of tumor spread are classified as the hepatic hilum type and the hepatic bed type (fig. 1) [6].

In the hepatic hilum type, a relatively small tumor in the gallbladder neck infiltrates the hepatic hilum and causes obstructive jaundice.

In the hepatic bed type, a large mass in the gallbladder fundus and body penetrates into the hepatic parenchyma through the gallbladder bed, often with contiguous spread to the gastrointestinal tract such as the colon, duodenum, and stomach.

In the combined type, a huge mass occupying the entire gallbladder involves both the gallbladder bed of the
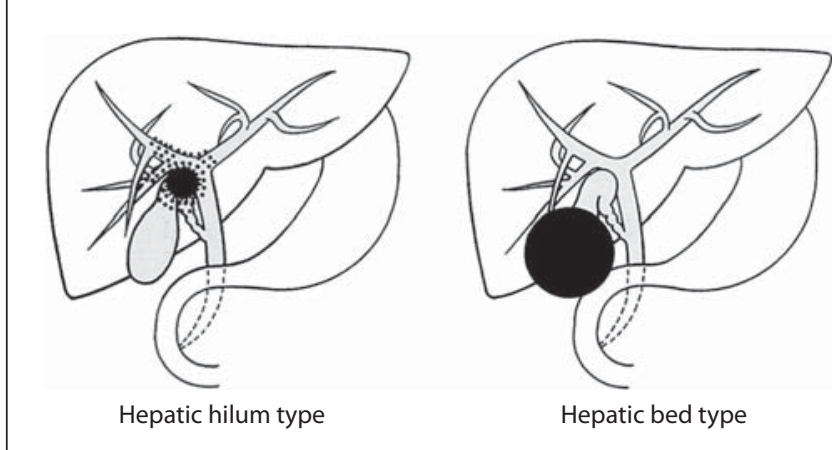

Fig. 1. Mode of tumor spread in advanced gallbladder cancer.

liver and the hepatic hilum. Contiguous gastrointestinal involvement is also common.

Prognosis depends on the stage rather than on the type of tumor spread. Postoperative long-term survival has been achieved even in patients with obstructive jaundice due to hilar hepatic invasion as far as distant metastasis is absent [2]. These types of gallbladder cancer can be diagnosed preoperatively by clinical and radiological examination. This information helps the surgeon plan surgical procedures with curative intent.

\section{Two Types of Hepatectomy for Advanced Gallbladder Cancer}

\section{Right Hepatectomy with Biliary Reconstruction}

In the hepatic hilum type or combined type, extended right hepatectomy and caudate lobectomy plus biliary reconstruction, often with portal vein reconstruction, are necessary just as in a curative procedure for hilar cholangiocarcinoma because the tumor extends into the hepatic hilum and/or right portal pedicle (fig. 2). Preoperative biliary decompression and portal vein embolization are recommended to prevent postoperative hepatic failure in patients with compromised functional reserve [7].

\section{Case 1}

A 66-year-old female presented with jaundice. Her serum level of CEA was remarkably elevated as $1,383 \mathrm{ng} /$ $\mathrm{ml}$. CT demonstrated that a large mass arising from the gallbladder invaded deeply into the liver (fig. 3). Cholangiography revealed bile duct obstruction at the hepatic hilum (fig. 3). A diagnosis was made of the combined type of advanced gallbladder cancer. 


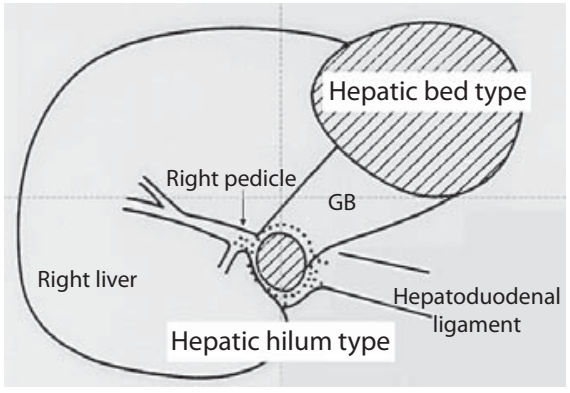

Fig. 2. Schematic illustration of hepatic involvement by advanced gallbladder cancer according to the type of tumor spread.

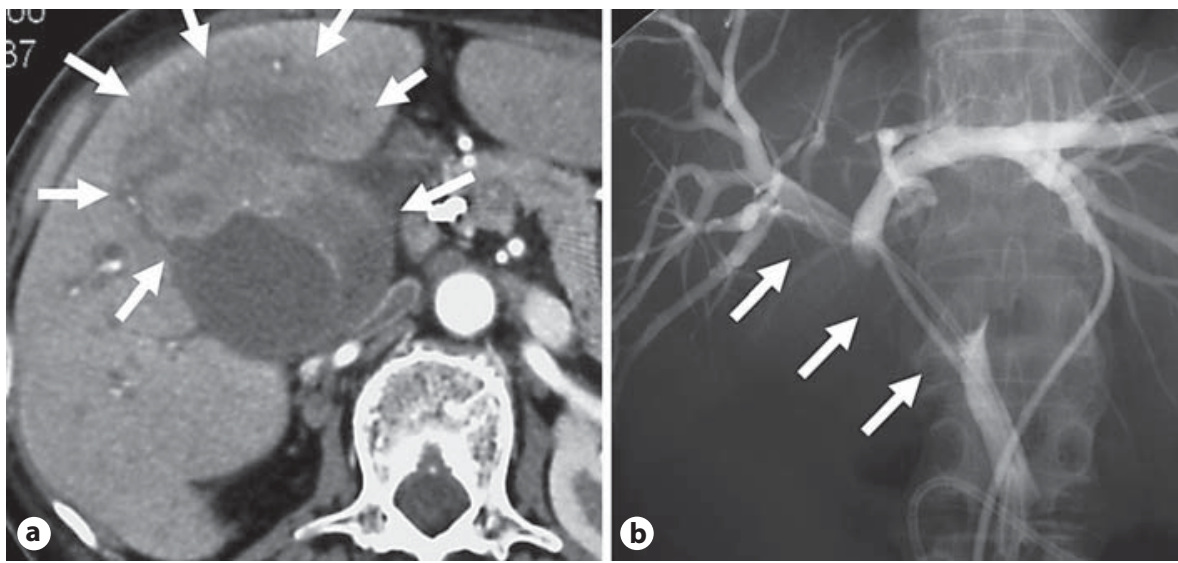

Fig. 3. Case 1. a CT demonstrating that a large mass arising from the gallbladder invades deeply into the liver (arrows). b Cholangiography through a percutaneous drainage catheter showing bile duct obstruction at the hepatic hilum (arrows).

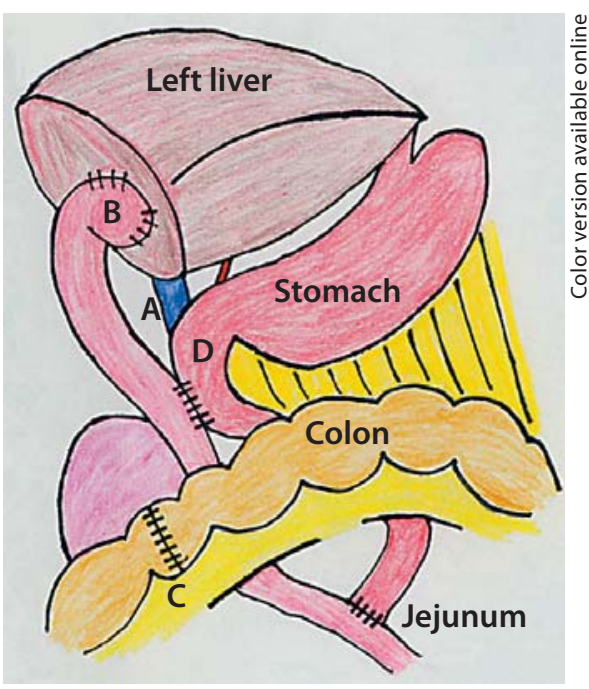

Fig. 4. Case 1. Schematic illustration showing reconstruction after extended right hepatectomy and right caudate lobectomy with concomitant resection of the bile duct, portal vein, duodenum, and colon. A: portal vein reconstruction by interposition of right external iliac vein graft; B: hepaticojejunostomy in a Roux-en-Y fashion; C: colocolostomy; D: patch repair of the duodenal defect in a side-to-side anastomosis fashion with the elevated jejunal limb.

\section{Operative Technique}

After confirming negative para-aortic disease by frozen section pathology, radical surgery was started. The involved colon was resected segmentally, and extended wedge resection was applied for the involved duodenum because it was considered similarly effective as an alternative to pancreatoduodenectomy [8]. The hepatic artery was divided just distal to the bifurcation with the gastroduodenal artery since the left hepatic artery was replaced from the left gastric artery. Following division of the common bile duct at the suprapancreatic level, systematic lymphadenectomy around the head of the pancreas was completed. The solely remaining skeletonized portal trunk was anastomosed to the left portal vein just beneath the umbilical portion with the interposed autovein graft harvested from the right external iliac vein. Preceding portal vein reconstruction a standard procedure of right hepatectomy with preceding division of the right portal vein was performed in combination with en bloc resection of the huge tumor involving the portal bifurcation $[9,10]$. Then, right hepatectomy was carried out with extension to the inferior part of the segment 4 (S4a) to ensure $1-\mathrm{cm}$ surgical margin adjacent to the tumor directly invading into the hepatic parenchyma. The reconstructive procedures are shown in figure 4.

The operative time was $13 \mathrm{~h}$ and $12 \mathrm{~min}$, and blood loss was $1,480 \mathrm{ml}$. No blood transfusion was needed. Postoperative course was uneventful. Pathological examination of the surgical specimen revealed well-differentiated tubular adenocarcinoma with T4N0M0, stage III [11].

One year later, three intraperitoneal disseminated nodules were resected. The patient is alive without disease recurrence 9 years after initial surgery. 

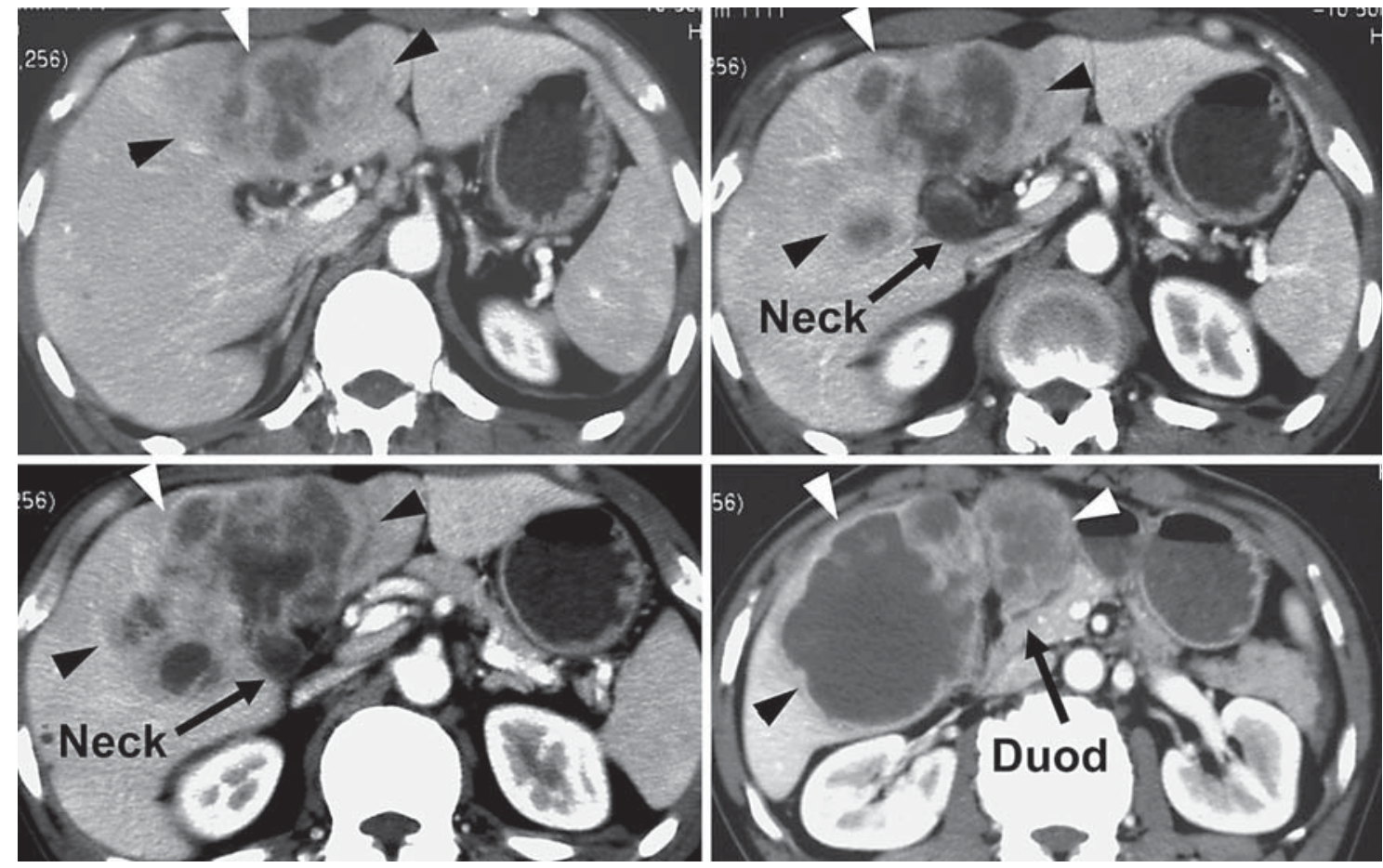

Fig. 5. Case 2. CT showing a 12-cm mass in S4a, 5, and 6 in the liver arising from the gallbladder fundus (arrowheads) and involving the proximal duodenum. Note the gallbladder neck is free from tumor involvement, suggesting no hilar or biliary invasion.

\section{Ventral Hepatectomy without Biliary Reconstruction}

In the hepatic bed type, the right portal pedicle can be basically preserved because even a huge tumor does not involve the hepatic hilum. The extent of hepatectomy is individualized from limited wedge resection to extended right hepatectomy based on the relationship between the tumor and the intrahepatic portal pedicles. Extended wedge hepatectomies include S4a (the inferior part of the segment 4) and S5 resection, S4a56 resection, and S3456 resection which is indicated when the top of the umbilical portion is involved. These can be called 'ventral hepatectomy' since hepatic parenchyma ventral to the hilar plate $[12]$ is resected. Systematic lymphadenectomy within the hepatoduodenal ligament and around the head of the pancreas can be completed with preservation of the common duct.

\section{Case 2}

A 57-year-old male visited a nearby hospital following annual health check disclosing abnormal serum levels of liver enzymes despite the absence of symptoms. Because a huge mass was detected on abdominal ultrasonography, he was referred to Hokkaido University Hospital. CT demonstrated a $12-\mathrm{cm}$ mass in the S4a, 5, and 6 in the liver arising from the gallbladder fundus and involving the proximal duodenum, although the gallbladder neck and biliary ductal system were free from tumor involvement (fig. 5). These findings were typically compatible with the hepatic bed type of advanced gallbladder cancer.

\section{Operative Technique}

Following a report of frozen section pathology proving negative para-aortic disease, radical surgery was started. The involved duodenum was resected as segmental gastroduodenectomy. After isolating and taping the hepatic arterial system, portal trunk, and common duct, systematic lymphadenectomy was completed around the head of the pancreas. The cystic duct was divided at the confluence with the common duct, and the cystic artery as well at its origin at the right hepatic artery. Along the right side of the umbilical portion of the left portal vein, several small pedicles of the S4a were divided at their origins; also the branches to S4a were divided preserving the S4 pedicle trunk and branches to S4b. Approaching 
Fig. 6. Case 2. Operative photo after ventral hepatectomy of S4a, 5, and 6. The S4 and $\mathrm{S} 4 \mathrm{~b}$ pedicle, right side of the umbilical plate, hilar plate, and right anterior portal pedicle (Rt ant pedicle) are exposed. Note the preserved common bile duct (CBD) and the right hepatic artery (Rt HA) running beyond it.

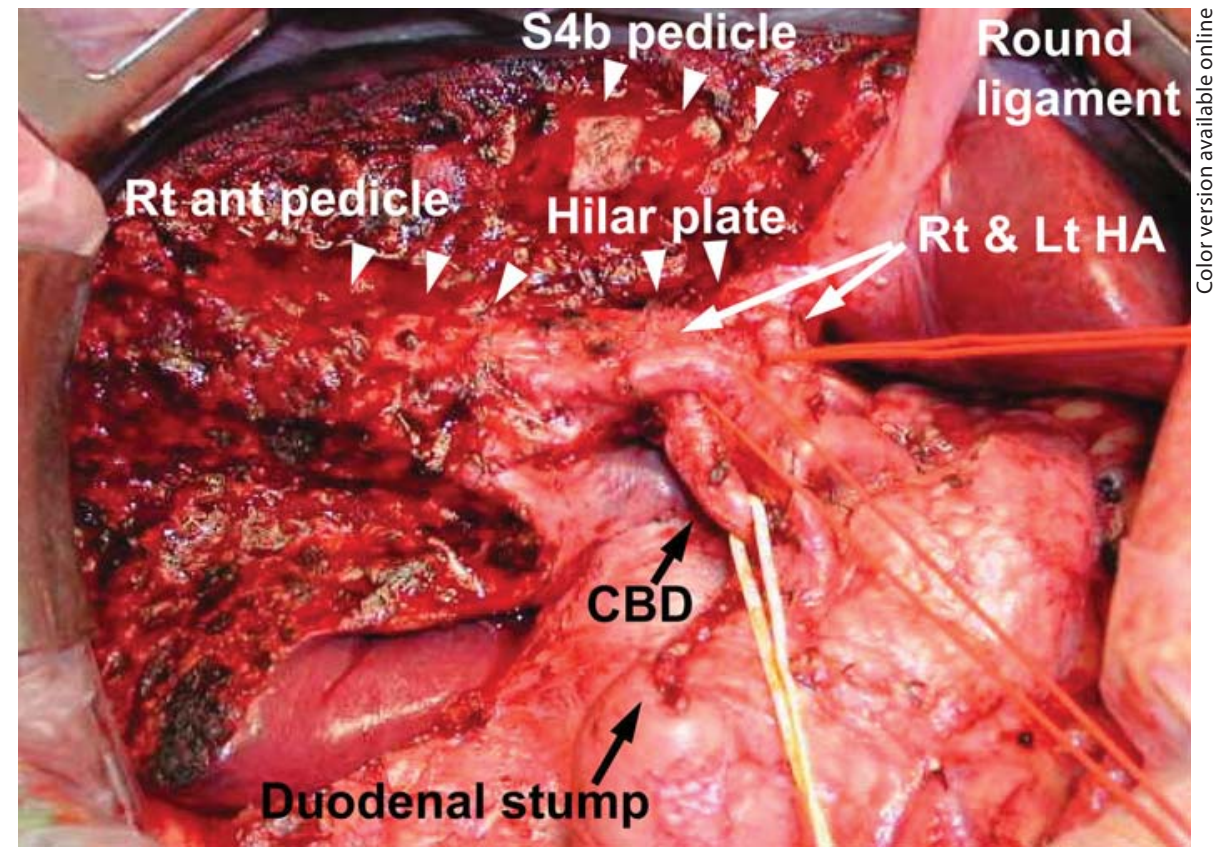

the hepatic hilum, the hilar plate and the anterior surface of the right anterior portal pedicle were exposed; then, the cystic plate [12] was transected at the confluence with the hilar plate. Along the right anterior portal pedicle, several branches to S5 were divided to ensure $1 \mathrm{~cm}$ or more surgical margin adjacent to the intrahepatic tumor. On the ventral surface of the liver as well, hepatic transection was performed with $1 \mathrm{~cm}$ or more margin, including the ischemic area due to pedicle branch division. The middle hepatic vein was divided in the hepatic transection plane. Finally, several pedicle branches to S6 were divided preserving the right posterior portal pedicle, and the ischemic area was transected. After completion of ventral hepatectomy, the $S 4$ and $S 4 \mathrm{~b}$ pedicle, right side of the umbilical plate [12], hilar plate, and right anterior portal pedicle were exposed (fig. 6). Gastrojejunostomy in a Billroth type II fashion was added.

The operative time was $8 \mathrm{~h}$ and $27 \mathrm{~min}$, and blood loss was $830 \mathrm{ml}$. No blood transfusion was needed. Postoperative course was uneventful. Pathological examination of the surgical specimen revealed undifferentiated carcinoma of spindle and giant cell type, partially with tubular adenocarcinoma and squamous cell cancer. The retroportal lymph node was positive, and the disease was staged as T4N1M0, stage III [11].

$\mathrm{He}$ is alive without disease recurrence 7 years after surgery.

\section{Disclosure Statement}

All authors certify that they have no commercial associations (e.g. consultancies, stock ownership, equity interest, patent/licensing arrangements, etc.) that might pose a conflict of interest in connection with the submitted article.

\section{References}

1 Nakamura S, Suzuki S, Konno H, Baba S, Muro H: Ten-year survival after hepatectomy for advanced gallbladder carcinoma: report of two cases. Surgery 1995;117:232-234.

2 Kondo S, Nimura Y, Kamiya J, Nagino M, Kanai M, Uesaka K, Yuasa N, Sano T, Hayakawa N: Five-year survivors after aggressive surgery for stage IV gallbladder cancer. J Hepatobiliary Pancreat Surg 2001;8:511-517.

3 Kondo S, Nimura Y, Hayakawa N, Kamiya J, Nagino M, Uesaka K: Extensive surgery for carcinoma of the gallbladder. Br J Surg 2002; 89:179-184.

4 Kondo S, Nimura Y, Hayakawa N, Kamiya J, Nagino M, Uesaka K: Regional and paraaortic lymphadenectomy in radical surgery for advanced gallbladder carcinoma. $\mathrm{Br}$ Surg 2000;87:418-422.

5 Noji T, Kondo S, Hirano S, Tanaka E, Ambo Y, Kawarada Y, Morikawa T: CT evaluation of paraaortic lymph node metastasis in patients with biliary cancer. J Gastroenterol 2005;40:739-743. 
6 Kondo S, Nimura Y, Kamiya J, Nagino M, Kanai M, Uesaka K, Hayakawa N: Mode of tumor spread and surgical strategy in gallbladder carcinoma. Langenbeck Arch Surg 2002;387:222-228.

7 Kondo S, Hirano S, Ambo Y, Tanaka E, Okushiba S, Morikawa T, Katoh H: Forty consecutive resections of hilar cholangiocarcinoma with no postoperative mortality and no positive ductal margins: results of a prospective study. Ann Surg 2004;240:95101.
8 Hirano S, Tanaka E, Shichinohe T, Saitoh K, Takeuchi M, Senmaru N, Suzuki O, Kondo S: Feasibility of en-bloc wedge resection of the pancreas and/or the duodenum as an alternative to pancreatoduodenectomy for advanced gallbladder cancer. J Hepatobiliary Pancreat Surg 2007;14:149-154.

9 Kondo S, Katoh H, Hirano S, Ambo Y, Tanaka E, Okushiba S: Portal vein resection and reconstruction prior to hepatic dissection during right hepatectomy and caudate lobectomy for hepatobiliary cancer. Br J Surg 2003;90:694-697.
10 Hirano S, Kondo S, Tanaka E, Shichinohe T, Tsuchikawa T, Kato K: No touch resection of hilar malignancies with right hepatectomy and routine portal reconstruction. J Hepatobiliary Pancreat Surg 2009; 16: 502-507.

11 International Union Against Cancer (UICC): TNM Classification of Malignant Tumors, ed 7. Oxford, Wiley-Blackwell, 2009.

12 Kawarada Y, Das BC, Taoka H: Anatomy of the hepatic hilar area: the plate system. J Hepatobiliary Pancreat Surg 2000;7:580586. 